Rev. Biol. Trop., 48(2/3): 689-702, 2000

www.ucr.ac.cr www.ots.ac.cr www.ots.duke.edu

\title{
Dieta estacional del Tayassu pecari (Artiodactyla: Tayassuidae) en el Parque Nacional Corcovado, Costa Rica
}

\author{
Mariana Altrichter ${ }^{1}$, Joel C. Sáenz ${ }^{1}$, Eduardo Carrillo ${ }^{1}$ y Todd k. Fuller ${ }^{2}$. \\ 1 Programa Regional en Manejo de Vida Silvestre para Mesoamérica y el Caribe, Universidad Nacional. \\ Apartado 1350-3000 Heredia, Costa Rica. Fax: 2377036, e-mail: jsaenz@una.ac.cr \\ 2 University of Massachussetts, Departament of Forestry and Wildlife Management. Amherst, MA 01003, EEUU.
}

Recibido 5-V-1999. Corregido 17-II-2000. Aceptado 8-III-2000.

\begin{abstract}
The diet of the white-lipped peccari Tayassu pecari was studied from July 1996 to April 1997 in Corcovado National Park, Costa Rica, through fecal analysis and direct observations. The feces consisted of $61.6 \%$ fruits, $37.5 \%$ vegetative parts, $0.4 \%$ invertebrates and $0.5 \%$ unidentified material. These proportions are similar to those reported for white-lipped peccaries diet in South America, but the species consumed were different. In Corcovado, the white-lipped peccary fed on parts of 57 plant species ( 37 of them fruits). Moraceae was the most represented family. In contrast, the diet of the Peruvian Amazon peccary primarily consists of plant parts (Arecaceae). Costa Rican peccary diet consisted of vegetative parts from Araceae and Heliconaceae. Direct observation showed that peccaries spent $30 \%$ of feeding time rooting. Samples taken from rooting sites suggest that peccaries fed on earthworms. Diet differed between months, seasons and habitats. They ate more fruits in coastal and primary forests and more vegetative parts in secondary forest. In the months Octubrer and November the consumption of vegetative parts exceeded fruit consumption.
\end{abstract}

Key words: Seasonal diet, fecal analysis, direct observations, Tayassu pecari, white-lipped peccary, rain forest, Costa Rica.

El chancho de monte o cariblanco (Tayassu pecari) es el mamífero terrestre grande más amenazado de extinción en todo el neotrópico, debido esencialmente a la pérdida de su hábitat natural y la presión de cacería (Emmons 1984, Smythe 1987). La presencia actual del chancho de monte es discontinua y fragmentada en la mayor parte de su rango de distribución, que se extiende desde el sur de México hasta el norte de Argentina (Sowls 1997, March 1990). Esta especie habita bosques tropicales húmedos, pero también está presente en sabanas y en bosques secos (Mayer \& Brandt 1982, Sowls
1997). A pesar de la importancia de su conservación y manejo, hay poca información acerca de su biología y ecología, y la mayoría son reportes descriptivos. Algunos estudios al respecto fueron hechos en Sudamérica (Kiltie 1981a; Terborgh \& Kiltie 1984; Bodmer 1989a, 1989b, 1990; Olmos 1993; Hernández et al. 1995; Barreto et al. 1997), mientras que en Centroamérica solo se conoce la distribución y estado de conservación de algunas poblaciones (Vaughan 1983; March 1990).

Varios estudios han caracterizado al chancho de monte como un omnívoro, incluyendo 
en su dieta frutos, plantas, lagartijas, anfibios, huevos de aves, tortugas, y carroña (Méndez 1970, Mayer \& Wetzel 1987); además, la creencia popular menciona que los chanchos de monte se alimentan entre otras cosas, de vertebrados pequeños, incluso serpientes venenosas. Bodmer (1989a) sugirió que el chancho de monte en la Amazonía peruana es especialmente frugívoro, siendo los frutos y semillas de palmas (Arecaceae) su principal recurso alimentario. En cambio en áreas xerófitas de Brasil y Venezuela la dieta del chancho de monte está constituida principalmente por raíces (Olmos 1993, Barreto et al. 1997).

Según Janzen (1983) y Terborgh (1992), en los bosques húmedos tropicales la producción de frutos en algunas especies varía ampliamente durante el año, por lo tanto, la dieta de un animal frugívoro como el chancho de monte debería variar también. Sin embargo, Bodmer (1990) no encontró variación en su dieta relacionada a las estaciones en la Amazonía.

La información sobre la dieta del chancho de monte es importante para el conocimiento de su historia natural y para comprender mejor sus interrelaciones dentro de la comunidad de ungulados. De igual manera, se requiere de esta información como base para futuras investigaciones sobre el papel que esta especie cumple en determinar la distribución, densidad y composición de comunidades de plantas en los bosques tropicales (Janzen 1974, Smythe 1987) y para su manejo y conservación en el país, en especial como especie amenazada.

Los objetivos del estudio fueron: a) determinar la composición de la dieta del chancho de monte en el Parque Nacional Corcovado y b) determinar la variación temporal y espacial en su dieta.

\section{MATERIALES Y MÉTODOS}

El estudio se realizó en el Parque Nacional Corcovado (PNC), península de Osa, sur de Costa Rica $\left(8^{\circ} 26^{\prime}-8^{\circ} 39^{\prime} \mathrm{N}\right.$ y $83^{\circ} 25^{\prime}$ $\left.83^{\circ} 45^{\prime} \mathrm{O}\right)$. El sitio de estudio cubrió aproxima- damente 3000 ha. La mayor parte del PNC se encuentra dentro de la zona de vida de "bosque muy húmedo tropical" (Hartshorn 1983). El clima es caliente y muy húmedo, con una época seca de diciembre a abril y una húmeda de mayo a noviembre. Los meses de máxima precipitación son octubre y noviembre $(>500 \mathrm{~mm}$ /mes) y los más secos febrero y marzo $(<100$ $\mathrm{mm} / \mathrm{mes}$ ). La temperatura media anual es de $26^{\circ} \mathrm{C}$ y la precipitación varía de $3800 \mathrm{~mm}$ anuales en las bajuras hasta $6500 \mathrm{~mm}$ en las zonas altas (Hartshorn 1983). La topografía del PNC presenta una llanura y una zona montañosa hasta $745 \mathrm{msnm}$. A gran escala, se pueden diferenciar bosques primarios y secundarios premontano y de bajura, asociación costanera, bosque de yolillo (Raphia taedigera) y pantano herbáceo (Naranjo 1995). Para mayor detalle sobre la vegetación ver Vaughan (1981) y Phillips (1993).

Los datos fueron recolectados de julio de 1996 a abril de 1997. En diciembre no se pudo observar los chanchos de monte porque abandonaron momentáneamente el área de estudio.

Ocho chanchos de monte (seis hembras y dos machos) pertenecientes a cuatro manadas (dos individuos por manada) diferentes fueron capturados y marcados con radiocollares (Carrillo et al. 1997). Utilizando la técnica de radiotelemetría se ubicaron y siguieron las manadas entre las 7:00 h y las 17:00 hr. Dos métodos fueron usados para estimar la dieta, la observación directa y el análisis de heces. Los datos siempre fueron recolectados solo por dos personas, con la intención de causar la menor perturbación al comportamiento natural de los animales

Estimación de dieta por observación directa: Se realizaron observaciones de los animales utilizando la técnica de barrido (scan sample) con registros instantáneos cada $5 \mathrm{~min}$, siempre con cuatro o más animales a la vista. Los datos fueron recolectados como tiempos dedicado a cada actividad de alimentación, según Martin y Bateson (1986), y clasificados en cuatro categorías: 1) Consumo de frutos: búsqueda de frutos con el hocico sobre el suelo caminando entre un fruto y otro, y el procesamiento del fruto en la boca; 2) Consumo de plantas: considerado desde 
que muerde una parte vegetativa de la planta hasta que la procesa en la boca; cada vez que se observó a un chancho de monte alimentándose de plantas se registró la especie y la parte consumida; 3) Consumo bajo tierra: actividad desarrollada por el chancho de monte cuando escarbaba en la tierra con las patas y el hocico, y 4) Consumo de materia animal: persecución y masticación de animales sobre el suelo.

Para conocer de que se alimentaron los animales al escarbar en la tierra, se tomó tres muestras de $500 \mathrm{~g}$ de tierra cada una en los sitios recientemente escarbados. Una muestra se obtuvo del centro del escarbadero, otra inmediatamente al lado, y otra alejada unos $10 \mathrm{~m}$ donde no estuviera escarbado, pero por donde los animales habían pasado. Se separó, lavó y pesó la materia animal encontrado en las muestras, y cuando fue posible se identificó hasta nivel de especie.

Estimación de dieta por análisis de heces: Se recolectaron un máximo de cinco heces por manada por día. Las heces se lavaron con agua y jabón para quitar la grasa y suciedad adherida, pasándolas por un colador de $1 \mathrm{~mm}$ y secadas al sol. Se utilizó la técnica de diez puntos (Korschgen 1987, McCoy et al. 1990) para obtener muestras al azar de cada excremento. Mediante una curva con los promedios (de una muestra de heces) acumulados de los componentes más comunes (fibra y fruto), se determinó un número de 50 muestras puntuales como óptimo para tener representada la variación de los componentes en cada excremento. Con un estereoscopio se identificaron cuatro categorías: 1) Fruto: semillas, restos de testas (cáscaras), de exocarpo y de endocarpo; 2 ) Parte vegetativa: material fibroso y restos de hojas, 3) Materia animal: los restos de exoesqueletos, mudas de larvas o pedazos de cuerpos de invertebrados y pequeños vertebrados 4) Desconocido: el material que no se pudo clasificar como ninguna de las categorías anteriores.

Clasificación de hábitats: Para determinar los tipos de hábitats donde los animales se alimentaban, se establecieron 37 transectos de $60 \mathrm{~m}$ x $10 \mathrm{~m}$ ubicados de forma sistemática cada 100 $\mathrm{m}$ ubicados de manera alternada en senderos es- tablecidos del PNC, donde se midieron tres variables: altura estimada del dosel, diámetro a la altura de pecho (DAP) y composición del sotobosque. A partir de éstas variables se definieron tres tipos de hábitats: a) bosque primario, con una altura del dosel de 20 a $40 \mathrm{~m}$ y árboles de diámetros mayores a $120 \mathrm{~cm}$ y sotobosque compuesto por palmas enanas; $b$ ) bosque secundario, con un dosel de 15 a $30 \mathrm{~m}$ de altura, DAPs no mayores a $65 \mathrm{~cm}$, sotobosque con áreas cubiertas de platanillos (Heliconia spp.) y c) bosque costero, de similar altura y DAPs que el primario pero con sotobosque abierto. La presencia de Geonoma spp. y platanillos son indicativos de bosque primario y secundario respectivamente (Janzen 1983).

Análisis de datos: Para cuantificar los elementos encontrados en la heces se utilizó la siguiente fórmula: $\mathrm{P}_{\mathrm{i}}=(\mathrm{T} / \mathrm{S}) * 100$ (Romero y Mandujano 1995).

Donde $\underline{P}_{\mathrm{j}}$ es el porcentaje del componente i, $\underline{T}$ es el número de puntos del componente $\underline{i}$ tocados por las agujas y $\underline{\mathrm{S}}$ es el número total de puntos (50).

Se distinguieron las heces del hábitat costero, porque durante la recolecta de datos los chanchos de monte permanecieron todo el día en dicho hábitat, por eso se supuso que estas heces representan lo consumido en este hábitat; además, la presencia en estas heces de restos de semillas de especies que solo crecen en el bosque costero confirmó esta suposición. En cambio no se pudo distinguir las heces encontradas en hábitats primario y secundario, debido al frecuente movimiento entre ambos hábitats en el mismo día. Por ésta razón se agrupó como heces de hábitat interior.

Se hicieron comparaciones entre hábitats, entre meses y entre estaciones para determinar la variación temporal y espacial en la dieta de los chanchos de monte del PNC. Se usaron pruebas estadísticas no paramétrica como Análisis de Varianza de una vía (Kruskal-Wallis) y pruebas para dos muestras (Mann-Whitney), en la mayoría de los comparaciones. Para detectar diferencias en la biomasa de la materia animal (lombrices) encontrada en los puntos de muestreo de los escarbaderos, se utilizó un Análisis de Varianza paramétrico de una vía (ANOVA). 


\section{RESULTADOS}

Los chanchos de monte consumieron frutos estacionalmente, mientras que las partes vegetativas de platanillos y las de la familia Araceae fueron utilizadas durante todo el período de estudio. El Cuadro 1 resume las especies consumidas con mayor frecuencia en cada mes (20\% de las observaciones diarias de cada mes). Se identificaron 57 especies vegetales consumidas por el chancho de monte, de los cuales 37 son frutos y semillas (Apéndice 1). Estas especies pertenecen a 24 familias, de las cuales Moraceae es la más común para los frutos y Araceae para las partes vegetativas, cuyas partes fueron generalmente hojas y tallos nuevos, con menor frecuencia flores y plántulas (Cuadro 2, Apéndice 1).

$\mathrm{Se}$ obtuvieron resultados similares entre las observaciones directas y los análisis de heces en cuanto al porcentaje de consumo de frutos, constituyendo un $62 \%$ de la dieta (Cuadro 3). Los frutos y partes vegetativas estuvieron presentes en más del $90 \%$ de las heces y que representaron alrededor del $99 \%$ de los componentes en las heces, por otro lado, los invertebrados y materia no identificable estuvieron presentes en las heces en un $0.4 \mathrm{y}$ $0.5 \%$ respectivamente .

En las muestras de tierra tomadas de los escarbaderos se encontraron larvas de insectos, isópodos y lombrices. La cantidad de larvas e isópodos fue muy baja, menor a 0.1 $\mathrm{g} / \mathrm{kg}$ de tierra, por lo tanto no se consideraron para los análisis. La especie de lombriz más abundante fue Pontoscolex corethurus (Glossoscolecidae). El peso promedio de las lombrices difirió significativamente entre los tres sitios de toma de muestras, siendo mayor adyacente al escarbadero ( $\mathrm{x}=2.10 \mathrm{~g} / \mathrm{kg}$ ) que en

\section{CUADRO 1}

Plantas consumidas (20\% de las observaciones mensuales) por el chancho de monte (Tayassu pecari) en el Parque Nacional Corcovado, de julio 1996-abril 1997. Los datos son de observación directa

TABLE 1

Eaten plants (20\% of the monthly observations) by White-lipped pecary (Tayassu pecari) in Corcovado National Park, July 1996-April 1997. Data provided are from direct observations.

\begin{tabular}{|c|c|c|c|c|c|c|c|c|c|}
\hline Plantas & Jul & Ago & Sep & Oct & $\begin{array}{l}\text { Meses } \\
\text { Nov }\end{array}$ & Ene & Feb & Mar & Abr \\
\hline Heliconia spp. & $\mathrm{X}$ & $\mathrm{X}$ & $\mathrm{X}$ & $\mathrm{X}$ & $\mathrm{X}$ & $\mathrm{X}$ & $\mathrm{X}$ & $\mathrm{X}$ & $\mathrm{X}$ \\
\hline Araceae & $\mathrm{X}$ & $\mathrm{X}$ & $\mathrm{X}$ & $\mathrm{X}$ & $\mathrm{X}$ & $\mathrm{X}$ & $\mathrm{X}$ & $\mathrm{X}$ & $\mathrm{X}$ \\
\hline Quararibea asterolepis & & $\mathrm{X}^{*}$ & $\mathrm{X}^{*}$ & & & & & & \\
\hline Spondias spp. & & $\mathrm{X}^{*}$ & $\mathrm{X}$ & & & & & $\mathrm{X}$ & \\
\hline Licania operculipetala & & $\mathrm{X}$ & $\mathrm{X}^{*}$ & $\mathrm{X}^{*}$ & & & & & \\
\hline Ficus spp. & $\mathrm{X}^{*}$ & $\mathrm{X}$ & & $\mathrm{X}^{*}$ & $\mathrm{X}^{*}$ & & $\mathrm{X}$ & & \\
\hline Brosimum spp. & & & & & & $\mathrm{X}^{*}$ & $\mathrm{X}^{*}$ & $\mathrm{X}^{*}$ & $\mathrm{X}^{*}$ \\
\hline Inga spp. & & & & & & & & $\mathrm{X}$ & $\mathrm{X}$ \\
\hline Anacardium excelsum & & & & & & & & $\mathrm{X}$ & $\mathrm{X}$ \\
\hline Astrocaryum spp. & & & & & & & & & $\mathrm{X}^{*}$ \\
\hline
\end{tabular}

$\left(^{*}\right)$ plantas más importantes en dieta en cada mes

$\left(^{*}\right)$ most important plants of the diet per month 


\section{CUADRO 2}

Proporción promedio(\%), frecuencia de ocurrencia de los componentes en las heces, y tiempo promedio (\%) dedicado a las actividades (técnica de barridos) de consumo del chancho de monte (Tayassu pecari), en el Parque Nacional Corcovado de Julio 1996 a abril 1997.

TABLE 2

Average proportion (\%), ocurrence frequency of the feces components, and feeding average time (\%) (scan sampling) of White-lipped pecary (Tayassu pecari) in Corcovado National Park,

July 1996-April 1997.

$\begin{array}{lccc}\text { Componente } & \text { Proporción(\%) } & \begin{array}{c}\text { Heces } \\ (\mathrm{n}=98) \\ \text { Ocurrencia(\%) }\end{array} & \begin{array}{c}\text { Barridos } \\ (\mathrm{n}=1715)\end{array} \\ \text { Tiempo(\%) }\end{array}$

CUADRO 3

Comparación del tiempo promedio(\%) dedicado por

los chanchos de monte (Tayassu pecari) a actividades de consumo de fruto, planta (partes vegetativas) y bajo tierra. Parque NacionalCorcovado, julio 1996-abril 1997.

TABLE 3

Comparison of average time (\%) dedicated by White-lipped pecary (Tayassu pecari) to feed on fruits, plants (vegetative parts) and rooting. Corcovado National Park, July 1996-April 1997.

$\begin{array}{cclll}\text { Primario (\%) } & \begin{array}{c}\text { Hábitat } \\ \text { Secundario (\%) }\end{array} & \text { Costero } & \mathrm{H} & \mathrm{p} \text { * } \\ & & & & \\ 23.8 & 27.9 & 32.6 & 6.3 & \mathrm{p}<.05 \\ 11.2 & 12.4 & 5.0 & 12.9 & \mathrm{P}<.01 \\ 65.0 & 59.7 & 62.3 & 1.2 & \mathrm{P}=0.59\end{array}$

\footnotetext{
* Kruskal-Wallis
} 
el centro del escarbadero ( $\mathrm{x}=1.16 \mathrm{~g} / \mathrm{kg}$ ) y en el sitio no escarbado $(\mathrm{x}=0.56 \mathrm{~g} / \mathrm{kg}$ ) (ANOVA $\mathrm{p}<.001$ ). Ni por observación directa ni en las heces se encontró evidencia de consumo de vertebrados.

Dieta entre hábitats: Se encontraron diferencias significativas entre el tiempo dedicado al consumo de frutos, siendo mayor en el hábitat costero que en los otros. Por otro lado, fue mayor el tiempo dedicado al consumo de plantas en el hábitat secundario que en los otros. El tiempo de consumo bajo tierra no difirió significativamente entre los hábitats (Cuadro 3). Considerando todo el período de estudio, hubo diferencias significativas entre los hábitats primario y secundario en el tiempo dedicado a las tres actividades de alimentación, siendo alto el consumo de frutos en el hábitat primario (Mann-Whitney $\mathrm{p}<.001$ ), mientras que el consumo de plantas y bajo tierra fue mayor en el hábitat secundario que en el primario (Mann-Whitney $\mathrm{p}<.001$ y $\mathrm{p}<$ .001 respectivamente) (Fig. 1).

El porcentaje de frutos encontrado en las heces durante el período que usaron los tres hábitats, presentó diferencias entre los hábitats

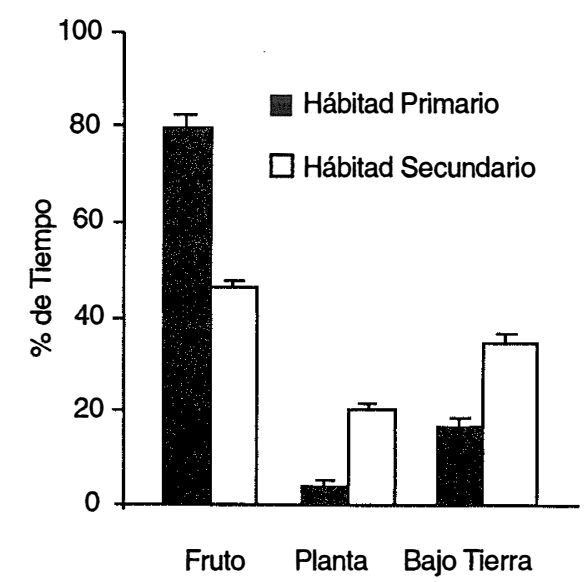

Fig. 1. Porcentaje promedio ( $\pm 1 E S$ ) de tiempo dedicado por Tayassu pecari al consumo de frutos, plantas y bajo tierra, en hábitats primario y secundario. Julio 1996-abril 1997.

Fig. 1. Mean percentage time ( $\pm 1 \mathrm{ES})$ spent by Tayassu pecari to feed on fruit, plants and rooting, in primary and secondary habitats. July 1996-april 1997. costero e interior, siendo mayor en el costero que en el interior (Mann-Whitney $\mathrm{p}<.01$ ). El porcentaje de materia vegetativa fue mayor en el hábitat interior, que en el costero (MannWhitney $\mathrm{p}<.001$ ), y no hubo diferencias significativas entre los hábitats en el porcentaje de materia animal.

Dieta Estacional: En los hábitats primarios y secundarios hubo diferencias significativas entre meses en el tiempo dedicado a las tres actividades de alimentación, mientras que en el hábitat costero fue significativa la variación sólo en el tiempo dedicado al consumo de plantas (Fig. 2A), aumentando el consumo en octubre (Kruskal-Wallis $\mathrm{p}<.05$ ). El consumo de frutos y bajo tierra entre los meses no presentó diferencias (Fig. $2 \mathrm{~A}$ ). El tiempo dedicado al consumo bajo tierra en el bosque secundario disminuyó a través del tiempo, mientras que los frutos fueron más consumidos en febrero y abril, pero muy poco mínimo en julío y enero (Kruskal-Wallis $\mathrm{p}<.001$ ). Las plantas constituyeron la mayor proporción con respecto a los otros items en el mes de enero (Kruskal-Wallis $\mathrm{p}<.001$ ) (Fig. 2 B).

El tiempo dedicado a consumir frutos en el bosque primario aumentó con el tiempo, alcanzando más del $80 \%$ de enero a abril (KruskalWallis $\mathrm{p}<.001)$, mientras que el consumo bajo tierra disminuyó inversamente (Kruskal-Wallis $\mathrm{p}$ $<.001$ ). La ingestión de plantas fue bajo durante los meses del estudio, con excepción de octubre donde el consumo de frutos y de plantas fueron semejantes (30\%) (Fig. 2 C). En mismo hábitat hubo diferencias en el consumo de frutos entre la época húmeda y la seca (Mann-Whitney $\mathrm{p}<$ .001 ), pero mayor en la época seca que en la húmeda. El tiempo dedicado a la alimentación con partes vegetativas y bajo tierra fue mayor en proporción en la época húmeda que en la seca (Mann-Whitney p <.001) (Fig. 2 C). En el hábitat secundario hubo diferencias en el tiempo dedicado al consumo de frutos durante la época seca y la húmeda (Mann-Whitney $\mathrm{p}<.001$ ).

El consumo de plantas fue mayor en la época seca que en la húmeda (Mann-Whitney $\mathrm{p}<.05$ ), y el consumo bajo tierra fue más alto en la época húmeda que en la seca (MannWhitney $\mathrm{p}<.001)$. 

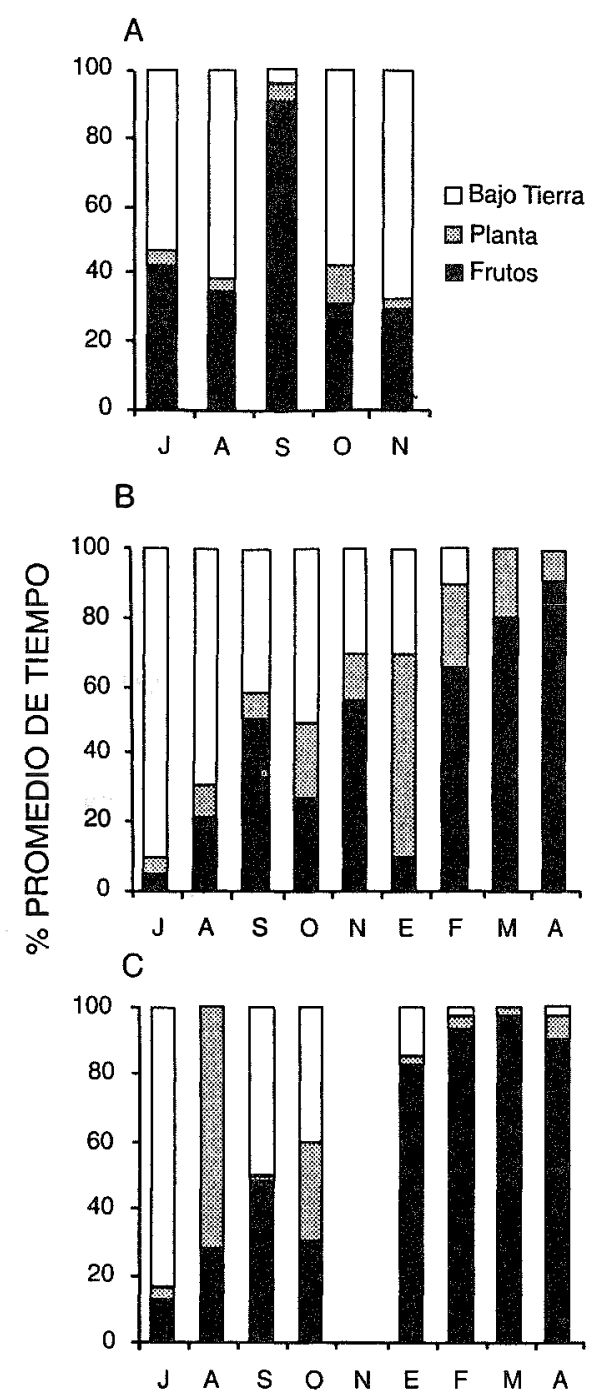

Fig. 2. Porcentajes promedios mensuales de tiempo dedicado por Tayassu pecari al consumo de frutos, plantas y bajo tierra en A) hábitat costero, B) hábitat secundario y C) hábitat primario. Julio 1996-Abril 1997.

Fig. 2. Mean percentage time spent by Tayassu pecari feeding on fruits, plants and rooting in A) coastal habitat, B) secondary habitat and C) primary habitat. July 1996-April 1997.

Los porcentajes de frutos, partes vegetativas y materia animal en las heces variaron a lo largo del período de estudio (Fig. 3). El porcentaje de frutos consumidos fue mayor en el mes de agosto y de enero a abril (Kruskal-Wallis $p$ $<.001)$ que en los otros meses. El consumo de las partes vegetativas fue mayor en octubre y

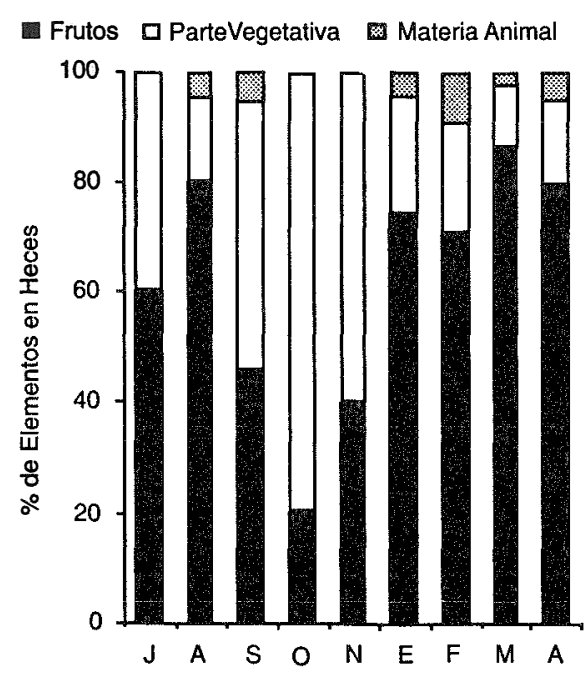

Fig. 3. Porcentaje promedio mensual de la proporción de ítemes (frutos, partes vegetativas y materia animal) en las heces de Tayassu pecari. Julio 1996-Abril 1997.

Fig. 3. Monthly percentaje food item average (fruits, vegetative plants and animal material) in Tayassu pecari feces. July 1996-April 1997.

noviembre, excediendo a los frutos (KruskalWallis $\mathrm{p}<.001)$. Un alto consumo de materia animal ocurrió en septiembre, febrero y abril (Kruskal-Wallis $\mathrm{p}<.01$ ) (Fig. 3). No se incluye datos del hábitat costero,porque el tamaño de muestras fue pequeño (10 heces).

\section{DISCUSION}

Métodos usados: Los porcentajes de frutos encontrados en las heces y el tiempo dedicado por los chanchos al consumo de frutos fueron similares. Los valores coinciden con lo reportado para análisis estomacales (Kiltie 1981a, Bodmer et al. 1997). Según Johnson y Wofford (1983), el uso de las heces es un método confiable para estimar dieta de hervíboros. Sin embargo, el consumo de lombrices no se vio reflejado en las heces encontradas en el PNC, posiblemente por la ausencia de quitina en las lombrices, mientras que sí es revelado 
por la observación directa. La técnica de barrido subestima las actividades menos conspicuas (Martin y Bateson 1986), lo cual sucedió con el tiempo dedicado a la alimentación de plantas, que es una actividad que no realiza todo el grupo en conjunto sino solo algunos individuos, no así, la actividad de alimentación bajo tierra que puede estar sobrestimada por las observaciones. En cambio la cantidad de fibra es sobre estimada en el análisis de las heces (DiMare 1994). El análisis de heces fue más sensible a los cambios en la dieta que la observación directa, mostrando mayor variación, pero las tendencias fueron las mismas, mostrando una buena complementaridad entre las dos técnicas.

Dieta: Las hojas y/o tallos, de platanillos y especies de la familia Araceae fueron consumidas durante todo el período de estudio. Las demás especies fueron consumidas en períodos cortos, con excepción de los Ficus spp., que parecen no tener un período restringido de fructificación (Quesada et al. 1997). Llama la atención que dentro de la familia Araceae se encuentran plantas muy tóxicas (Escobar 1972), algunas de las cuales como Dieffenbachia spp. y Philodendron spp., son consumidas por los chanchos. Las hojas y tallos tiene altas cantidades de compuestos estructurales que los vertebrados no pueden digerir si no es por medio de fermentación bacteriana (Owen 1980), lo cual es una adaptación evolutiva del género Tayassu (Sowls 1997).

En los bosques húmedos de la Amazonía peruana, además de las palmas (Arecaceae), las especies más consumidas fueron de las familias Sapotaceae y Menispermaceae (Bodmer et al. 1997), mientras que en zonas de bosques secos las especies más importantes fueron guácimo (Guazuma ulmifolia), jobo (Spondias mombin ), laurel (Cordia spp.) y Prosopis spp (Hernández et al 1995, Sowls 1997). En el PNC las especie más consumidas fueron las de la Moraceae. Esto ilustra la amplitud de la dieta de esta especie, respondiendo a la diferente composición florística o abundancia de ciertos frutos de los diversos bosques tropicales. Las palmas constituyen el principal recurso alimentario para el chancho en la Amazonía (Kiltie y Terborgh 1983, Terborgh 1986, 1992, Bodmer 1989a, 1989b).
En este estudio, a diferencia de los anteriores las palmas no parecen ser un recurso importante para los chanchos de monte, a pesar de ser plantas abundantes en el PNC (Sáenz y Carrillo, en pre.). Solo en marzo y abril se observó a los animales alimentarse de palmas principalmente de semillas de coquito (Astrocaryum spp.). Sin embargo, para 1998 la manadas de chanchos forrajearon intensamente semillas de coquito y viscoyol (Bactris spp.) durante la época seca (J. Sáenz com. pers.). Las semillas de las palmas contienen entre $50 \%$ y $65 \%$ de grasas, pero tienen poca proteína (Owen 1980), por lo tanto, es probable que los chanchos de monte obtienen el suplemento necesario de grasas de otras especies, que no requieran tanto esfuerzo de procesamiento como las semillas duras de palmas (Kiltie 1981b, 1982), como por ejemplo Carapa guianensis que contiene $60.5 \%$ de grasa (López 1999).

La alimentación de semillas duras de palmas es un recurso adaptativo del chancho de monte para evitar la competencia con otros ungulados frugívoros (Kiltie 1982, Bodmer 1989a, 1989b, Terborgh 1992). Sin embargo, en el PNC las dantas (Tapirus bairdii) comen muy pocos frutos de palmas (Naranjo 1995) y el otro competidor potencial es el saíno (Tayassu tajacu), pero su densidad poblacional en el PNC es baja comparada con los chanchos de monte (J. Sáenz com. pers.). Esto indicaría que el chancho de monte no tiene una fuerte competencia inter-específica con otros ungulados de similar dieta y roedores que remueven y depredan las semillas de estas palmas. Por estas razones, las interrelaciones en la comunidad de ungulados en el PNC podrían tener características muy particulares.

El $62 \%$ de consumo de frutos y $38 \%$ de partes vegetativas encontrado en las heces coincide con lo reportado por Kiltie (1981a) y Bodmer et al. (1997) mediante análisis de contenidos estomacales, mientras que la proporción y el porcentaje de ocurrencia de materia animal en las heces fue mucho menor a lo que encontraron Kiltie (1981a) y Bodmer et al. (1997). Estos mismos autores y otros (Méndez 1970, Sowls 19.97) mencionan a vertebrados como parte de la dieta de los chanchos de mon- 
te, pero en este estudio no hubo indicios de consumo de vertebrados.

El alto porcentaje de tiempo dedicado a la búsqueda bajo tierra sugiere que allí encuentran un recurso importante. Se descarta la posibilidad que estén comiendo solo raíces, porque no se encontraron restos en las heces, aunque tallos y raíces son muy importantes para chanchos de monte en Brasil y Venezuela (Olmos 1993, Barreto et al. 1997). Las raíces tienen un porcentaje de digestibilidad menor que las hojas, tallos y frutos (Bodmer et al. 1997), por lo tanto, la probabilidad de detectar restos en las heces es alta. Los chanchos escarban en busca de semillas (Kiltie 1981c), pero el PNC no se encontraron semillas en las muestras de tierra de los escarbaderos sino lombrices.

El consumo de lombrices podría ser una estrategia de los chanchos en el PNC para compensar la limitación de alimentos ricos en proteínas. Skinner et al. (1976) tomaron muestras de tierra de escarbaderos de cerdos salvajes (Potamochoerus porcus) y encontraron mayor proporción de lombrices que de otros posibles alimentos (larvas o raíces). Por otro lado, Diong (1982) encontró lombrices en el $90 \%$ de los estómagos de Sus scrofa en un bosque húmedo tropical de Hawaii. La especie de lombriz de Hawaii es la misma que hay en el PNC, la cual es la más abundante y común (P. Lapied, com. pers.). Scott y Pelton (1975), sugieren que la importancia de las lombrices como alimento se subestima porque la masticación y digestión es muy rápida, y es difícil detectarlas aún en análisis de estómagos frescos. Por este motivo posiblemente no se ha reportado que el consumo de lombrices sea tan importante para los chanchos como parece sugerir este estudio.

Bodmer (1990) no encontró variación en la dieta del chancho de monte relacionada a las estaciones, en este estudio se encontró grandes variaciones dietarias en el consumo de frutos y partes vegetativas, tanto entre los meses como entre las estaciones húmeda y seca. En cambio la materia animal encontrada en las heces no mostró una tendencia estacional evidente. Estos cambios en la dieta podrían estar relaciona. dos con fluctuaciones de la disponibilidad de frutos, la cual varía mucho a través del año en los bosques tropicales (Janzen 1983, Terborgh 1992, Altrichter et al. en pre.).

La variación espacial de la dieta durante la época húmeda en el hábitat costero se debió principalmente al consumo de semillas de camarón (Licania operculipetala), especie que crece solo en este tipo de ambiente (Quesada et al. 1997). En cambio durante el mismo período en el bosque interior se alimentaron mayormente de higos (Ficus spp.), garrochos (Quararibea asterolepis) y jobos. En ese mismo bosque fue muy alto el consumo de partes vegetativas, sobre todo tallos nuevos de platanillos, los cuales crecen en claros o en bosques secundarios. En un parque nacional como Corcovado se espera que un futuro el bosque se regenere completamente, lo que implicaría una disminución de platanillos. Ahora sabemos que esta planta ocupa un lugar importante en la dieta del chancho de monte. Análisis nutricionales de esta planta señalan que tiene bajo contenido de grasa $(1.98 \%)$, pero mayor cantidad de proteína cruda $(8.4 \%)$ que especies con alto contenido de grasa y energía bruta como coquito y vicoyol (López 1999). Sin embargo, habría que estudiar cuales son las requerimiento alimenticios de está especie, para estimar el posible efecto que causaría sobre los chanchos una disminución en la disponibilidad de heliconias en el PNC.

\section{AGRADECIMIENTOS}

Agradecemos al Programa Regional en Manejo de Vida Silvestre de la Universidad Nacional, Costa Rica, Parque Nacional Corcovado, Wildlife Conservation Society, Fondo Mundial para la Naturaleza (WWF), National Geographic Society y Ammonite Ltd., por financiar está investigación. A Carlos Drews y María T. Fernández por comentarios y revisión de este manuscrito. A Omar Laquis por su ayuda en el trabajo de campo. José González, Reinaldo Aguilar y Mike Grayum de INBio identificaron los frutos y plantas consumidos por los chanchos de monte. 


\section{RESUMEN}

Se estudió la dieta del chancho de monte (Tayassu pecari) por medio de análisis de heces y observación directa de julio de 1996 a abril de 1997 en el Parque Nacional Corcovado, Costa Rica. En las heces se encontró $61.6 \%$ de frutos, $37.5 \%$ de materia vegetativa, $0.4 \%$ de invertebrados y $0.5 \%$ no fue identificado. Estas proporciones coincidieron con lo reportado para Sudamérica, pero las especies consumidas fueron diferentes, sumando en total 57 especies, de los cuales 37 fueron de frutos. La familia más representada en la dieta fue Moraceae en lugar de Arecaceae como en la Amazonía peruana. Las partes vegetativas de la dieta pertenecieron principalmente a las familias Araceae y Heliconaceae. Mediante la observación directa se determinó un $30 \%$ del tiempo de alimentación dedicado a escarbar bajo tierra, lo cual es sugerido por el indicar que estarían alimentándose principalmente de lombrices, lo cual es sugerido por el análisis de muestras de los escarbaderos. Se encontraron variaciones temporales y espaciales en su dieta. Hubo diferencias en la dieta entre hábitats, entre meses, y entre estaciones (húmeda y seca). Los chanchos de monte se alimentaron más de frutos en los bosques costero y primario y más de plantas en el bosque secundario; sin embargo, el consumo de partes vegetativas superó al de frutos en los meses de octubre y noviembre.

\section{REFERENCIAS}

Barreto G. R., O. E. Hernández \& J. Ojasti. 1997. Diet of peccaries (Tayassu tajacu and T. pecari) in dry forest of Venezuela. J. Zool. Lond. 241: 279-284.

Bodmer, R., E. 1989a. Frugivory in Amazon ungulates. Ph.D. Thesis. University of Cambridge. Cambridge, UK. 234 p.

Bodmer, R., E. 1989b. Frugivory in Amazonian Artiodactyla: evidence for the evolution of the ruminant stomach. J. Zool. Lond. 219: 457-467.

Bodmer, R., E. 1990. Responses of ungulates to seasonal inundations in the Amazon floodplain. J. Trop. Ecol. 6: 191-201.

Bodmer, R. E., R. Aquino, P.E. Puertas, C.J. Reyes, T.G. Fang \& N.L. Gottdenker. 1997. Manejo y Uso Sustentable de Pecaríes en la Amazonía Peruana. Occassional. Paper of the IUCN Species Survival Commision No. 18. Ecuador y Secretaría CITES, Ginebra, Suiza. 102 p.
Carrillo, E., J. C. Saenz, T. K. Fuller \& M. Altrichter. 1997. Home range and activity patterns of white-lipped peccaries (Tayassu pecari) in Corcovado National Park. Symposium and Annual Meeting, Tropical diversity origins, maintenance, and Conservation. Organization for Tropical Studies, San José, Costa Rica. 124 p.

DiMare, M., I. 1994. Hábitos alimentarios del venado cola blanca en la Isla San Lucas, Puntarenas, Costa Rica, p. 73-90. In C. Vaughan and M. Rodríguez (eds.). Ecología y manejo del venado cola blanca en México y Costa Rica. EUNA, Heredia, Costa Rica.

Diong, Ch., H. 1982. Population biology and managment of the feral pig Sus scrofa in Qipahulu Valley, Maui Hawaii. Ph.D. Thesis, University of Hawaii, Hawaii.

Emmons, L., H. 1984. Geographic variation in densities and diversities on non-flying mammals in Amazonia. Biotropica 16: 210-222.

Escobar, N., A. 1972. Flora tóxica de Panamá. EUPAN, Panamá. 235 p.

Hartshorn, G., S. 1983. Plants. P. 118-157. In D. H. Janzen (ed.). Costa Rican Natural History, University of Chicago.

Hernández, O. E., G. R. Barreto, \& J. Ojasti. 1595. Observations of behavioural patterns of White-lipped peccaries in the wild. Mammalia 59: 146-148.

Janzen, D., H. 1974. Tropical blackwater rivers, animals and mast fruiting by the Dipterocarpaceae. Biotropica 6: 69-103.

Janzen, D., H.1983. Costa Rican Natural History. The University of Chicago.

Johnson, M., Q. \& H. Wofford. 1983. Digestion and fragmentation: influence on herbivore diet analysis. J. Wildl. Manage.47: 877-879.

Kiltie, R., A. 1981a. Stomach contents of rain forest peccaries (Tayassu tajacu and T. pecari). Biotropica 13: 234- 235. 
Kiltie, R., A. 1981b. The function of interlocking canines in rain forest peccaries (Tayassuidae). J. Mammalia. 62: 459-469.

Kiltie, R., A. 1981c. Distribution of palm fruits on a rain forest floor: why white-lipped peccaries forage near objects?. Biotropica 13:141-145.

Kiltie, R., A. 1982. Bite force as basis for niche differentation between rain forest peccaries (Tayassutajacu and T. pecari), Biotropica 14: 188-195.

Kiltie, R., A. y J. Terborgh. 1983. Observations on the behavior of rain forest peccaries (Tayassu pecari) in Perú: Why do White-lipped peccaries form herds?. Z. Tierpsychol 62:214-217.

Korschgen, L., J. 1987. Procedimientos para el análisis de los hábitos alimentarios, p. 119-133. In R. R. Tarrés (ed.). Manual de técnicas de gestión de vida silvestre. The Wildlife Society, Maryland, USA.

López, M., T. 1999. Apectos nutricionales de la dieta del chancho de monte (Tayassu pecari) en Parque Nacional Corcovado, Costa Rica. Tesis M.Sc. Universidad Nacional, Heredia, Costa Rica. 85 p.

March, I., J. 1990. Evaluación de hábitat y situación actual del pecarí de labios blancos (Tayassu pecari) en Mexico. Tesis M.Sc. Universidad Nacional, Heredia, Costa Rica. 285 p.

Martin, P. \& P. Bateson. 1986. Measuring behaviour, an introductory guide. Cambridge University Press. Cambridge. 213 p.

Mayer, J., J. \& P. N. Brandt. 1982. Identity, distribution and natural history of the peccaries, Tayassuidae, $\mathrm{p}$. 433-455. In Mares, M. A., and H. H. Genoways (Eds.). Mammalian biology in South America. Special Publication Series Pymatuning Laboratory of Ecology. Univ. of Pittsburgh, Pennsylvania.

Mayer, J., J. \& R. M. Wetzel. 1987. Tayassu pecari. Mammalian Species 293: 1-7.

McCoy, M. B., C. Vaughan, M. A. Rodríguez \& D. Kitchen 1990. Seasonal movement, home range, activity and diet of collared peccaries (Tayassu tajacu) in Costa Rican dry forest. Vida Silvestre Neotropical 2: 6-20.
Méndez, E. 1970. Los principales mamíferos silvestres de Panamá. Zool. Lab. Conmemorativo Gorgas. Panamá. 187 p.

Naranjo, E., P. 1995. Hábitos de alimentación del tapir (Tapirus bairdii) en un bosque tropical húmedo de Costa Rica. Vida Silvestre Neotropical 4(1):32-37.

Olmos, F. 1993. Diet of sympatric Brazilian caatinga peccaries (Tayassu tajacu and T. pecari) J. Trop. Ecol. 9: 255-258.

Owen, J. 1980. Feeding Strategy. The University of Chicago Press. Chicago.

Phillips, P., R. 1993. Key to vegetation types for the Osa Península, Costa Rica. Center for Space Research, University of Texas, Austin, Texas. USA.

Quesada, F. J., Q. Jiménez, N. Zamora, R. Aguilar \& J. González. 1997. Árboles de la Península de Osa. Instituto Nacional de Biodiversidad, Heredia, Costa Rica.

Romero, L., E. \& S. Mandujano. 1995. Hábitos almentarios del pecarí de collar (Pecari tajacu) en un bosque tropical caducifolio de Jalisco, México. Acta Zool. Mex. 64: 1-20.

Scott, D., Ch. \& M. R. Pelton. 1975: Seasonal food habits of the european wild hog in the Great Smoky Mountains National Park. Annu. Conf. Southeast. Assoc. Game and Fish Comm. 29: 585-593.

Skinner, J. D., G. J. Breytenbach \& the Late C.T.A. Maberly. 1976. Observations on the ecology and biology of the bushpig (Potamochoerus porcus Linn.) in the Northern Transvaal. S. Afr. J. Wildl. Res. 6: 123-128.

Smythe, N. 1987. The importance of mammals in neotropical forest management, p. 135-152. In J. C. F. Colon (ed.). Management of the forests of tropical america: prospects and technologies. U.S.D.A.Forest Service, Rio Piedras, Puerto Rico.

Sowls, L., K. 1997. Javelinas and other Peccaries: Their, Biology, Management, and Use. The Texas A\&M Univertsity Press. College Station. 321 p. 
Terborgh, J. 1992. Diversity and the tropical rain forest. Scientific American Library. Nueva York.

Terborgh,J. \& R. A. Kiltie. 1984. Ecology and behaviour of rainforest peccaries in Southern Peru. National Geographic Society, Research Reports. 17: 873-882.

Terborgh, J. 1986. Comunity aspects of frugivory in tropical forests, p. 1371-1384. In Estrada, A.y T. H. Fleming (eds.). Frugivores and seed dispersal. Netherlands: Dr. W. Junk Publisherts T:VS 15.
Vaughan, C. 1981. Parque Nacional Corcovado. Plan de manejo y desarrollo. EUNA. Heredia, Costa Rica. 351 p.

Vaughan, C. 1983. A report on dense forest habitat for endangered widlife species in Costa Rica. Environmental Sciences School, National University, Heredia, Costa Rica. 37 p.

Wetzel, R. \& J.W. Lovett. 1974. A collection of mammals from the Chacó of Paraguay. Univ. Connecticut. Ocass. Papers, Biol. Sci. Ser., 2:203-16.

\title{
APENDICE 1
}

Lista de plantas en la dieta del chancho de monte (Tayassu pecari) en el Parque Nacional Corcovado, Julio 1996-abril 1997. Partes comidas de cada planta: F: fruto, H: hoja, T: tallo, FL: flor, S: semilla, PL: plántula.

\begin{abstract}
APPENDIX 1
List of White-lipped pecary (Tayassu pecari) diet plants in Corcovado National Park, July 1996-April 1997. Eaten plant parts: F: fruit, H: leave, T: sprout, FL: flower, $S$ : seed, $P L$ : seedling
\end{abstract}

Especie

Anacardium excelsum

Anthurium spp.

Astrocaryum spp.

Bactris spp.

Brosimum alicastrum

Brosimum costaricarum

Brosimum utile

Carlodovica drudei

Cyclanthus spp.

Cissus spp.

Clarisia biflora

Compsoneura sprucei

Costus spp.

Crysophila guagara

Denpropanax spp.

Dieffenbachia concinna

Dieffenbachia oerstedii

Dieffenbachia spp.

Dieffenbachia pluricostratum

Ficus zarzalensis

Ficus maxima

Ficus spp.

Garcinia spp.

Geonoma spp.

Geonoma cuneata

Guarea spp.
Familia

Parte

Anacardiaceae

Arecaceae

Arecaceae

Moraceae

Moraceae

Moraceae

Cyclanthaceae

Cyclanthaceae

Vitaceae

Moraceae

Myristicaceae

Costaceae

Arecaceae

Araliaceae

Araceae

Araceae

Araceae

Araceae

Moraceae

Moraceae

Moraceae

Clusiaceae

Arecaceae

Arecaceae

Meliaceae

\section{F}

Araceae H,T,FL

$\mathrm{S}$

$S$

$\mathrm{F}$

S,PL

S,PL

$\mathrm{T}$

$\mathrm{T}$

F

F

$\mathrm{S}$

H

S

$\mathrm{F}$

$\mathrm{H}, \mathrm{T}$

$\mathrm{H}, \mathrm{T}$

$\mathrm{H}, \mathrm{T}$

$\mathrm{H}, \mathrm{T}$

$\mathrm{F}$

F

F

FL

FL

FL 
Heliconia spp.

Heliconaceae $\mathrm{T}$

Homalomena wendlandi

Aracaceae

T

Inga spp.

Iriartea deltoidea

Fabaceae

$\mathrm{H}, \mathrm{T}$

Licania operculipetala

Arecaceae

$S$

Licania platypus

Chrysobalanaceae

S,F

Mansoa spp.

Marantes panamensis*

Chrysobalanaceae

Bignoniaceae

$\mathrm{S}, \mathrm{F}$

Maripa nicaraguensis*

Chrysobalanaceae

Convolvulaceae

$\mathrm{S}, \mathrm{F}$

Monstera membranacea

Araceae

$\mathrm{H}$

F

Monstera spp.

Moutabea longifolia

Mussa acuminata

Polygalaceae

H,T

Otoba novagranate

$\mathrm{H}, \mathrm{T}$

Musaceae

$\mathrm{H}$

Myristicaceae

$\mathrm{F}, \mathrm{T}$

Parinari parvifolia

Chrysobalanaceae

Familia

Especie

Sapindaceae

Parte

Paullinia spp.

Philodendron auriculatum

Araceae

F

Philodendron inaequilaterum

Areceae

$\mathrm{H}, \mathrm{T}$

Philodendron platypetiolatum

Araceae

$\mathrm{H}, \mathrm{T}$

Pouteria spp.

Psidium guajaba

Quararibea asterolopis

Sapotaceae

Myrtaceae

Bombacaceae

Scheelea rostrata

Serjania spp.

Spathiphyllum spp.

Spondias mombin

Spondias radlkoferi

Arecaceae

Sapindaceae

Araceae

Anacardiaceae

Anacardiaceae

Araceae

Araceae

Urticaceae

Syngonium hastiferum

Urera spp.

Myristicaceae

* Uncertain 